\title{
Distribuição espaço-temporal da raiva bovina em Minas Gerais, 1998 a 2006
}

[Space and time distribution of bovine rabies in Minas Gerais, 1998 to 2006]

\author{
F.L. Menezes ${ }^{1,4}$, J.A. Silva ${ }^{2 *}$, E.C. Moreira ${ }^{2}$, J.N.C. Meneses ${ }^{2}$, D.F. Magalhães ${ }^{3}$, \\ A.D. Barbosa ${ }^{1,4}$, C.S.F. Oliveira, \\ ${ }^{1}$ Aluna de graduação - EV-UFMG - Belo Horizonte, MG \\ ${ }^{2}$ Escola de Veterinária - UFMG \\ Caixa Postal 567 \\ 30123-970 - Belo Horizonte, MG \\ ${ }^{3}$ Aluna de pós-graduação - EV-UFMG - Belo Horizonte, MG \\ ${ }^{4}$ Bolsista de Iniciação Científica
}

\begin{abstract}
RESUMO
Estudou-se a situação epidemiológica da raiva bovina em Minas Gerais de 1998 a 2006. Foram avaliadas 6873 fichas de diagnóstico de raiva por imunofluorescência direta. Para análise da distribuição temporal da raiva, foram construídas tabelas e gráficos no software Excel 2003 e o mapeamento dos diagnósticos foi feito no aplicativo Tabwin 1.4. Verificou-se tendência anual decrescente do número de exames e dos diagnósticos positivos $(Y=-41,133 x+544,89)$. Os meses de maio, junho e julho apresentaram o maior percentual de diagnósticos positivos. $\mathrm{O}$ número de municípios com raiva bovina variou ao longo do período estudado e manteve tendência decrescente $(Y=-7,0833 x+166,64)$, com expansão da doença para os municípios da região do Triângulo Mineiro.
\end{abstract}

Palavras-chave: bovino, raiva, epidemiologia, distribuição temporal, distribuição espacial

\begin{abstract}
Epidemiological data of bovine rabies were evaluated after analyzing 6,873 files collected in Minas Gerais state, from 1998 to 2006. Direct Immunofluorescence was performed to diagnosis the disease. The temporal distribution of the disease was analyzed after tables and graphics were made using the software Excel 2003. A mapping diagnosis was built using the applicatory Tabwin 1.4. It was observed an annual decreasing tendency in the number positive diagnosis $(Y=-41.133 x+544.89)$. A higher percentage of positive diagnosis for rabies was observed from May to July. There was a variation in the number of counties in which bovine rabies was diagnosed during the studied period and it maintained a decreasing trend $(Y=-7.0833 x+166.64)$, with expansion of the disease in direction of "Triangulo Mineiro" counties.
\end{abstract}

Keywords: bovine, rabies, epidemiology, temporal distribution, spatial distribution

\section{INTRODUÇ̃̃O}

A raiva, além de ser uma zoonose importante para a saúde pública, ocasiona grandes perdas econômicas para a pecuária. De acordo com Reis et al. (2003), a doença mata 100.000 bovinos por ano na América Latina e gera perdas de cerca de 30 milhões de dólares. No Brasil, estima-se uma perda de 15 milhões de dólares por ano com a morte de cerca de 40 mil bovinos acometidos pela doença (Bredt, 1996).

Em 1998, a raiva foi constatada em 428 municípios brasileiros, com registros de 1.150 exames positivos em bovinos naquele ano (Raiva..., 1998).

Este estudo dá continuidade à análise da distribuição temporal e espacial da raiva bovina,

Recebido em 4 de outubro de 2007

Aceito em 6 de junho de 2008

* Autor para correspondência (corresponding author)

E-mail: jasilva@vet.ufmg.br 
de 1976 a 1997, realizada por Silva et al., (2001), em Minas Gerais, tendo como referencial teórico a organização do espaço agrário, e considera as condições sociais, econômicas e políticas ocorridas nesse período.

O objetivo deste trabalho foi analisar a distribuição espacial e temporal da raiva bovina, em Minas Gerais, de 1998 a 2006.

\section{MATERIAL E MÉTODOS}

O espaço estudado foi o estado de Minas Gerais, constituído por 853 municípios e 1535 distritos, localizado na zona tropical do país, com uma estação climática seca e fria e outra quente e chuvosa, variando entre as diversas regiões do estado. As condições ambientais e a sua posição geográfica favorecem a existência de diversas espécies de quirópteros (Pacheco, 2005).

O rebanho bovino de Minas Gerais, segundo o IBGE para o ano de 2005 (Censo..., 2006), era de 21.403 .680 cabeças, o que corresponde a $10,3 \%$ do rebanho bovino nacional, que é constituído por 207.156.696 cabeças.

Para descrição da distribuição da raiva bovina nos municípios de Minas Gerais foram analisadas fichas contendo informações sobre os resultados de exames realizados pelos laboratórios do Centro de Controle de Zoonoses (CCZ) da Prefeitura Municipal de Belo Horizonte e do Instituto Mineiro da Agropecuária (IMA). Os diagnósticos foram realizados pela técnica de imunofluorescência direta no período referente a janeiro de 1998 a dezembro de 2006.

Elaborou-se um banco de dados, no qual foram registrados, para cada exame, o município de origem do animal, a instituição responsável pela realização do exame, o diagnóstico e a data (mês e ano). Os dados dessa série temporal equivalem aos números de diagnósticos de raiva bovina por mês, ano e municípios atingidos pela doença e foram processados no software EPIData 3.1.

A distribuição temporal da raiva bovina foi representada por tabelas e estimativas de tendência. As informações foram estruturadas no programa Excel versão 2003. Para elaboração dos mapas e a visualização da evolução da doença foi utilizado o software Tabwin versão 1.4 .

\section{RESULTADO}

No período de janeiro de 1998 a dezembro de 2006, foram realizados 6873 exames dos quais $84(1,2 \%)$ feitos no CCZ e $6789(98,8 \%)$ no IMA. Entre o período de 2002 e 2004, o laboratório do $\mathrm{CCZ}$ encontrava-se fechado para reformas, o que esclarece a não realização dos exames por esta instituição. Ao analisar o período estudado, verifica-se diminuição no número de exames (Tab. 1).

Do total de exames realizados, 3053 (44,42\%) foram positivos e $3820(55,58 \%)$ negativos (Tab. 1). Nos dois primeiros anos analisados o percentual de positivos foi maior que o de negativos com inversão a partir do ano de 2000.

O número de municípios positivos variou ao longo do espaço temporal de análise sendo superior aos negativos até 2000, com inversão até 2004 e aumento de municípios positivos em 2005 e 2006 (Tab. 2). No ano de 2002, os resultados foram iguais. Verifica-se que em 1998, dos 853 municípios do estado, 291 realizaram exames para doença. Desse total, $180(21,10 \%)$ apresentaram diagnósticos positivos para a raiva bovina. Em 2006, dos 199 municípios que realizaram exames, 116 registraram a doença, o que corresponde a $13,59 \%$ de positividade.

Em relação à distribuição da raiva ao longo do ano, verifica-se que os meses de maio, junho e julho apresentaram os maiores percentuais de resultados positivos (Tab. 3 ).

Conforme linha de tendência e equação da reta (Fig. 1) verifica-se que houve decréscimo de 41,13 diagnósticos positivos de raiva bovina ao ano, com coeficiente linear $\mathrm{R}^{2}=0,75$ e $\mathrm{P}=0,002$.

A reta de tendência mostra diminuição de $7,1 \%$ dos municípios com raiva bovina, ou seja, 61 desses deixaram de ter diagnóstico de raiva a cada ano. Tal estimativa é marginalmente significativa, pois a reta de tendência apresenta coeficiente linear $\mathrm{R}^{2}=0,43 \mathrm{e}$ $\mathrm{P}=0,052$, (Fig. 2).

A distribuição espacial da raiva bovina, avaliada anualmente nos 853 municípios, entre 1998 e 2006, com a propagação da doença para distintas regiões de Minas Gerais pode ser observada na Fig. 3. 
Tabela 1. Exames de imunofluorescência direta, para diagnóstico de raiva bovina, realizados pelo Centro de Controle de Zoonoses (CCZ) e Instituto Mineiro de Agropecuária (IMA), em Minas Gerais, 1998 a 2006

\begin{tabular}{|c|c|c|c|c|c|c|c|c|}
\hline \multirow{3}{*}{ Ano } & \multicolumn{8}{|c|}{ Exames } \\
\hline & \multicolumn{4}{|c|}{ Número } & \multicolumn{4}{|c|}{ Resultados } \\
\hline & $\mathrm{CCZ}$ & IMA & Total & $\%$ & Positivo & $\%$ & Negativo & $\%$ \\
\hline 1998 & 17 & 1069 & 1086 & 15,80 & 586 & 53,95 & 500 & 46,05 \\
\hline 1999 & 23 & 918 & 941 & 13,70 & 476 & 50,58 & 465 & 49,42 \\
\hline 2000 & 15 & 969 & 984 & 8,61 & 443 & 45,02 & 541 & 54,98 \\
\hline 2001 & 7 & 585 & 592 & 14,31 & 246 & 41,55 & 346 & 58,45 \\
\hline 2002 & $\ldots$ & 717 & 717 & 10,43 & 306 & 42,67 & 411 & 53,33 \\
\hline 2003 & $\ldots$ & 715 & 715 & 10,40 & 261 & 36,5 & 454 & 63,5 \\
\hline 2004 & $\ldots$ & 625 & 625 & 9,10 & 248 & 39,68 & 377 & 60,32 \\
\hline 2005 & 5 & 610 & 615 & 8,94 & 269 & 43,73 & 346 & 56,27 \\
\hline 2006 & 17 & 581 & 598 & 8,70 & 218 & 36,45 & 380 & 63,54 \\
\hline Total & 84 & 6789 & 6873 & 100 & 3053 & 44,42 & 3820 & 55,58 \\
\hline
\end{tabular}

Tabela 2. Municípios positivos e negativos para raiva bovina em Minas Gerais, 1998 a 2006

\begin{tabular}{cccccc}
\hline \multirow{2}{*}{ Ano } & \multicolumn{5}{c}{ Município } \\
\cline { 2 - 6 } & Positivo & $\%$ & Negativo & $\%$ & Sem informação \\
\hline 1998 & 180 & 21,10 & 111 & 13,01 & 562 \\
1999 & 167 & 19,57 & 104 & 12,19 & 582 \\
2000 & 150 & 17,58 & 104 & 12,19 & 598 \\
2001 & 90 & 10,55 & 119 & 13,95 & 644 \\
2002 & 118 & 13,83 & 118 & 13,83 & 617 \\
2003 & 127 & 14,88 & 132 & 15,47 & 594 \\
2004 & 104 & 12,19 & 115 & 13,48 & 634 \\
2005 & 129 & 15,12 & 107 & 12,54 & 617 \\
2006 & 116 & 13,59 & 83 & 9,73 & 654 \\
\hline
\end{tabular}

Tabela 3. Diagnóstico de raiva bovina, por mês, em Minas Gerais, 1998 a 2006

\begin{tabular}{lcccccc}
\multicolumn{1}{c}{ Mês } & Examinado & Negativo & Positivo & $\begin{array}{c}\text { \% Positivos } \\
\text { no mês }\end{array}$ & $\begin{array}{c}\text { \% Positivos } \\
\text { no total de } \\
\text { examinados }\end{array}$ & $\begin{array}{c}\text { \% Positivos } \\
\text { no total de } \\
\text { positivos }\end{array}$ \\
\hline Janeiro & 577 & 322 & 255 & 44,19 & 3,71 & 8,35 \\
Fevereiro & 507 & 285 & 222 & 43,78 & 3,23 & 7,27 \\
Março & 618 & 347 & 271 & 43,85 & 3,94 & 8,87 \\
Abril & 472 & 257 & 215 & 45,55 & 3,12 & 7,04 \\
Maio & 542 & 296 & 246 & 45,38 & 3,57 & 8,05 \\
Junho & 652 & 342 & 310 & 47,54 & 4,51 & 10,15 \\
Julho & 639 & 329 & 310 & 48,51 & 4,51 & 10,15 \\
Agosto & 649 & 374 & 275 & 42,37 & 4,0 & 9,0 \\
Setembro & 557 & 312 & 245 & 43,98 & 3,56 & 8,02 \\
Outubro & 534 & 296 & 238 & 44,56 & 3,46 & 7,79 \\
Novembro & 5933 & 345 & 248 & 41,82 & 3,6 & 8,12 \\
Dezembro & 533 & 315 & 218 & 40,90 & 3,17 & 7,14 \\
Total & 6873 & 3820 & 3053 & 44,42 & 44,42 & - \\
\hline
\end{tabular}




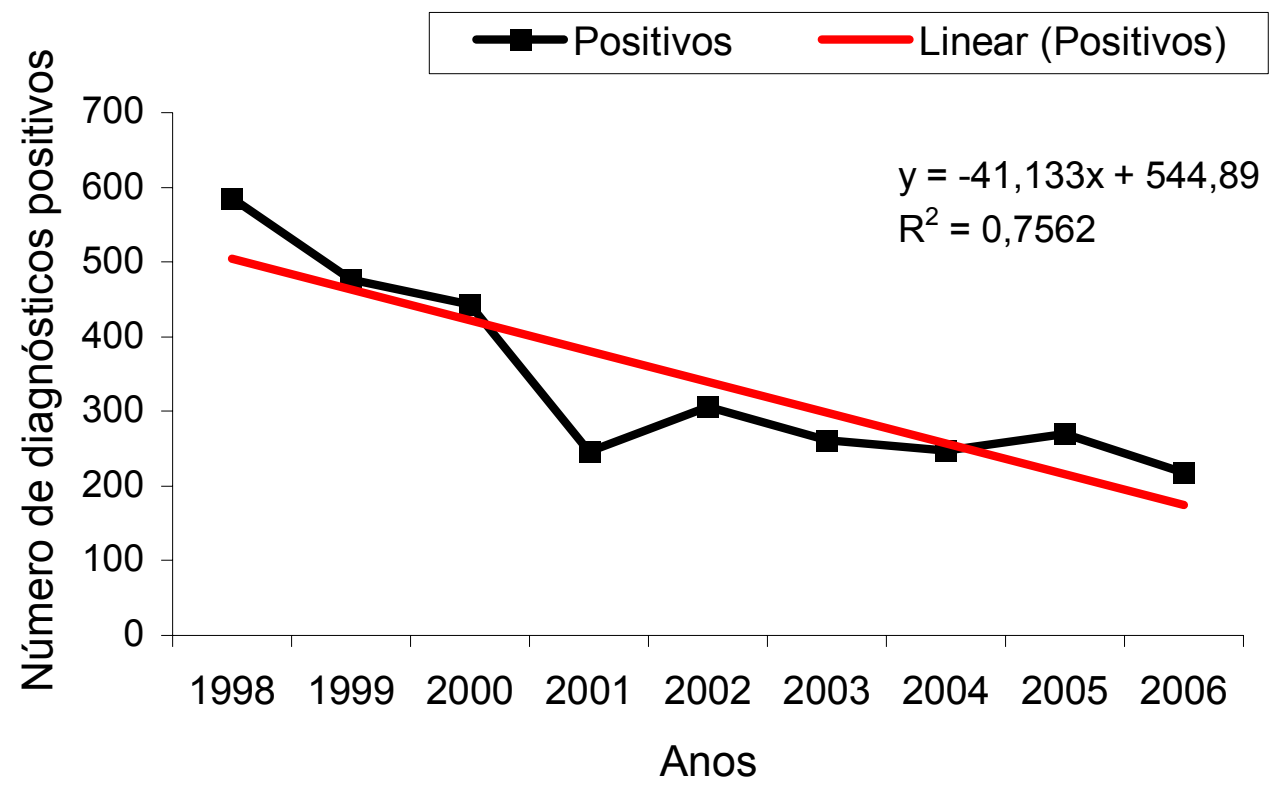

Figura 1. Tendência de diagnósticos positivos para raiva bovina em Minas Gerais, 1998 a 2006.

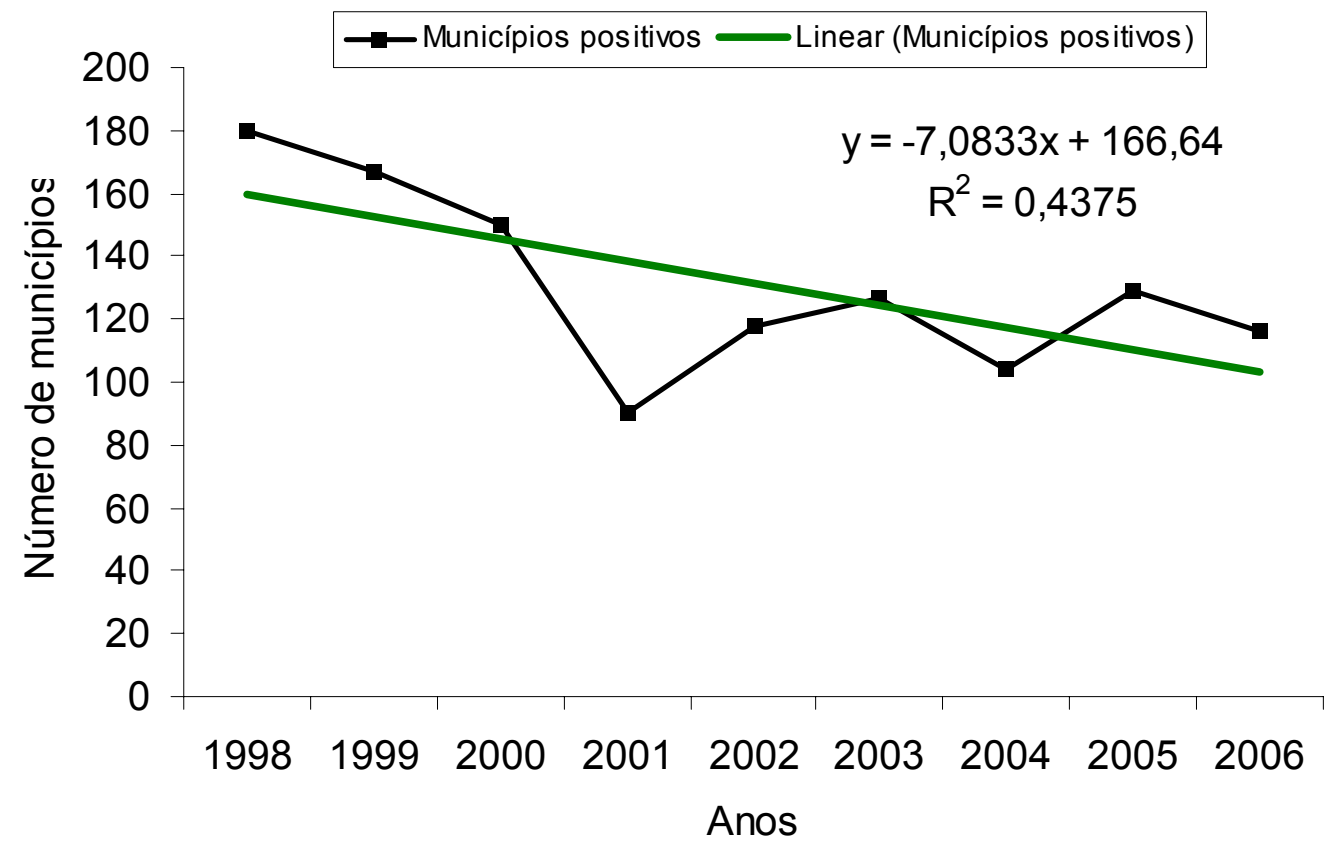

Figura 2. Tendência de municípios positivos para raiva bovina, em Minas Gerais, 1998 a 2006. 


\section{Menezes et al.}

1998

Negativos: 111

Sem informação: 582

Positivos: 180

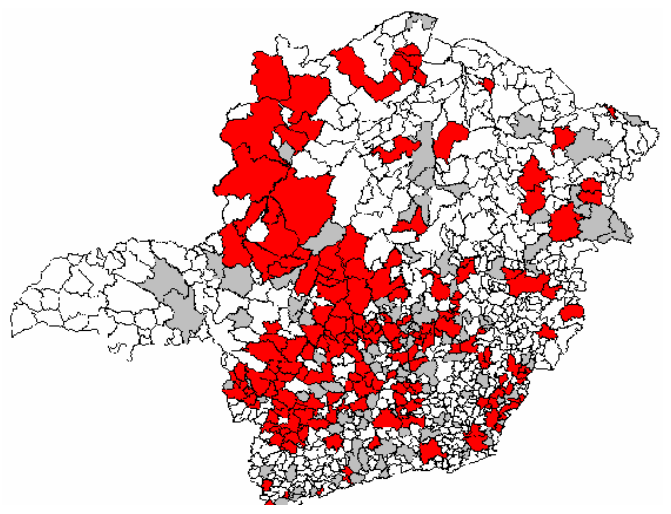

2003

Negativos: 132

Sem informação: 594

Positivos: 127
2001

Negativos: 119

Sem informação: 644

Positivos: 90

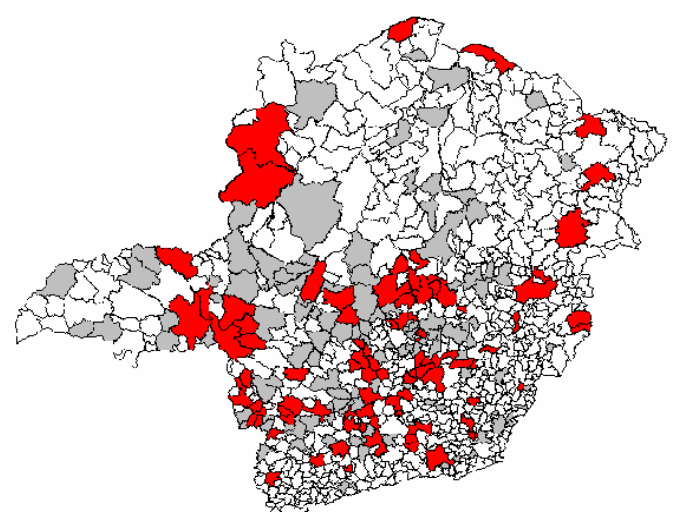

2006

Negativos: 83

Sem informação: 654

Positivos: 116
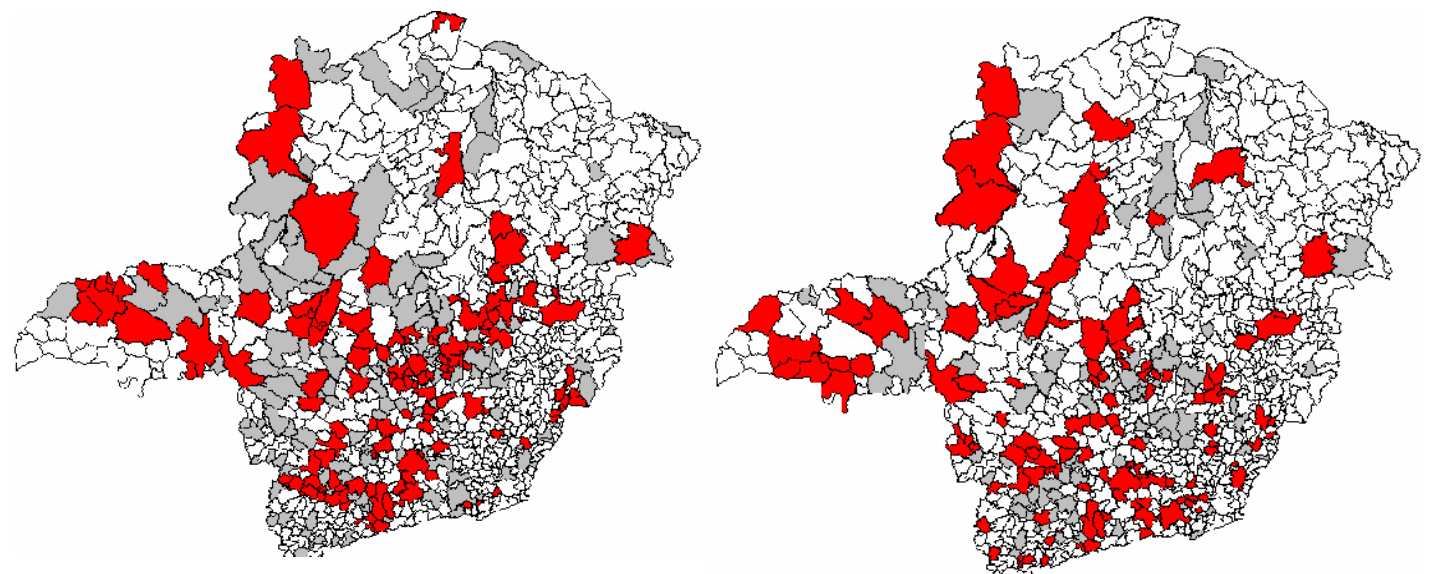

Figura 3. Distribuição espacial da raiva bovina nos municípios de Minas Gerais, 1998 a 2006.

\section{DISCUSSÃO E CONCLUSÕES}

O número decrescente de exames de raiva bovina realizados no estado pelos dois órgãos oficiais de 1998 a 2006 pode estar relacionado à diminuição dos casos clínicos da doença, aumento da utilização de vacinas anti-rábicas e programas de captura e tratamento de morcegos hematófagos com pasta anticoagulante realizados pelo IMA.

É importante ressaltar que a diminuição do número de exames de raiva pode estar também, relacionada ao não envio de material para laboratórios por parte dos veterinários. Essa 
prática baseia-se no fato de os profissionais diagnosticarem clinicamente a doença pela experiência de resultados positivos em anos anteriores, bem como a observação da presença de morcegos hematófagos na região, dispensando o diagnóstico confirmatório.

Segundo o percentual de resultados, houve tendência decrescente para diagnósticos positivos, o que aponta para a melhoria do controle da raiva bovina e a redução da freqüência da doença. Entretanto, deve-se ressaltar que esse número pode estar subestimado, pois se sabe que é comum na rotina dos veterinários de campo enviar amostras de apenas um bovino morto que tenha apresentado os sintomas da doença. Esse fato já foi assinalado por Luz (1988) e Silva (1993). Segundo Silva (1999), a subnotificação tornou-se institucionalizada e, assim, dificulta a análise mais precisa da situação epidemiológica da doença, especialmente quanto às perdas reais do efetivo bovino.

Conforme Valente e Amaral (1972), para cada caso diagnosticado de raiva existem, em média, seis animais mortos. Conforme os dados da Tab. 1, em 1998 foi possível estimar a perda de 3.516 (586 x 6) bovinos, número que se reduziu para $1.308(218 \times 6)$ bovinos acometidos pela doença em 2006.

Nos estudos epidemiológicos sobre raiva bovina, Luz (1988), Silva (1999) e Jayme (2003) observaram tendência crescente de diagnósticos positivos da doença nos estados de Minas Gerais e de Goiás. Os estudos demonstram a situação da doença nas décadas de 70, 80 e início dos anos 90 , quando se iniciaram os primeiros programas de combate à raiva no país. Os autores relatam o aumento do número de exames de raiva bovina, fato que pode ser relacionado à melhoria do sistema de vigilância epidemiológica, à ampliação do atendimento veterinário de campo e ao aprimoramento de laboratórios para a realização de exames pelos órgãos governamentais.

Verifica-se que os resultados coincidem com as descrições de Arellano-Sota (1988) e Tadei (1996) que relataram significativa redução do número de casos positivos de raiva bovina no Brasil e no estado de São Paulo, respectivamente. Tais observações do comportamento epidemiológico da doença podem estar relacionadas à eficiência dos programas de controle estabelecidos anteriormente.

A partir do ano 2000, o número de diagnósticos negativos foi superior ao de positivos. O aumento de resultados negativos sugere que outras encefalites de sinais clínicos similares estão ocorrendo em bovinos de Minas Gerais, o que também foi verificado por Silva (1999).

No período de abril a julho, concentrou-se o maior percentual de diagnósticos positivos. Nos demais meses, houve significativa variação dos diagnósticos. Um aspecto a ser avaliado é que o número de diagnósticos laboratoriais não reflete a ocorrência real do número de bovinos acometidos pela doença, sendo, portanto, insuficiente para afirmar sua possível sazonalidade, conforme já salientado por Silva et al. (2001). Esses autores sugeriram a necessidade de estudos mais aprofundados quanto à sazonalidade da raiva bovina no estado, considerando todos os casos reais de raiva ocorridos nos bovinos ao longo dos meses do ano.

A distribuição espacial da raiva bovina, descrita de acordo com o ano de ocorrência, mostra que o número de municípios positivos diminuiu ao longo do período em estudo, sendo que em alguns anos esse número foi inferior ao dos municípios sem casos da doença. É importante ressaltar que o número de municípios sem informações sobre raiva aumentou a partir de 2004, o que acarreta no desconhecimento da situação da doença nessas regiões.

Os municípios negativos apresentaram número crescente entre 2000 e 2004. Estes dados podem sugerir a melhoria do combate à doença nestas localidades e, assim, explicar a diminuição de envio de amostras para exames. Alguns municípios declarados negativos em tal período apresentaram, posteriormente, casos positivos de raiva bovina. Essa observação sugere que as medidas de controle e de vigilância da doença nessas áreas devem ser intensificadas.

A análise da dispersão da raiva bovina para diferentes regiões do estado mostra que, em 1998, a doença foi registrada nas regiões Sul e Sudoeste de Minas, Campo das Vertentes, Zona 
da Mata, Vale do Rio Doce, Mucuri, Jequitinhonha, Norte, Noroeste, Alto do Paranaíba, Oeste, Central, e Metropolitana de Belo Horizonte. Nesse ano, observa-se a presença intensa da doença nas regiões Noroeste, Alto do Paranaíba, Oeste, Sul e Sudeste, o que denota certa continuidade espacial dos casos de raiva. $\mathrm{O}$ aumento de diagnósticos positivos a partir de 2005 coincide com a expansão da raiva bovina para vários municípios da região do Triângulo Mineiro. Nessa região, observa-se expansão da doença a partir de 1999, com aumento do número de municípios positivos. Em 2001, registra-se a diminuição de municípios positivos no estado, onde em 90 deles $(10,6 \%)$ foram diagnosticados casos de raiva bovina. A diminuição dos casos, nas regiões mais afetadas anteriormente, pode ser devida ao uso de medidas preventivas aplicadas no combate à doença, tais como, a realização de vacinações nos rebanhos e o controle da população de morcegos hematófagos. Em 2003, o número de municípios negativos foi maior que o de positivos. A doença estava distribuída por todo estado e com ampliação para municípios vizinhos na região do Triângulo Mineiro. Em 2006, nota-se novamente número crescente de municípios positivos, $116(13,6 \%)$. Nesse ano, a raiva já havia acometido inúmeros municípios do Triângulo Mineiro em direção aos estados de Goiás e São Paulo.

Pela análise da distribuição espaço-temporal verificou-se a regularidade da presença da raiva bovina nos municípios de Minas Gerais, com maior ou menor intensidade nas diversas regiões, o que também já foi assinalado por Silva et al, 2001.

Os períodos de diminuição ou de expansão espacial da raiva bovina, provavelmente, devemse às medidas de combate aplicadas e às modificações da natureza pelo homem que implicam na coexistência de condições epidemiológicas diversas na população de morcegos. Isso pode explicar a evolução intermitente e a continuidade do endemismo da raiva no período de 1998 a 2006.

Assim, o combate à raiva bovina não deve ser limitado à sua confirmação na região, mas deve atuar de forma preventiva, incluindo a participação dos produtores, para conter a disseminação da doença nos rebanhos, tendo em vista o considerável efetivo de bovinos que favorecem a presença de morcegos hematófagos em todo o espaço de Minas Gerais.

\section{REFERÊNCIAS BIBLIOGRÁFICAS}

\section{ANUÁRIO ESTATÍSTICO DE MINAS GERAIS. v.9, p.44-218, 2000-2001.}

ARRELANO-SOTA, C. Vampire battransmitted rabies in cattle. Vet. Infect. Dis., v.10, p.707-709, 1988.

RAIVA animal. Bol. Def. Sanit. Anim., v.30, p.63-68, 1998

BREDT, A. ARAÚJO, F.A.A., JÚNIOR, C.J. et al. Morcegos em áreas urbanas e rurais: manual de manejo e controle. Brasília: Funasa, 1996. $175 \mathrm{p}$.

CENSO Agropecuário $2006 \quad$ - dados preliminares. disponível em: $<$ http://www.ibge.gov.br/estadosat/perfil.php?sig la=mg\#>. Acessado em: 20 jul. 2007.

JAYME, V.S. A modificação do espaço agrário e a dinâmica da raiva bovina em Goiás, Brasil, 1970-2001. 2003. 264f. Tese (Doutorado) Escola de Veterinária, Universidade Federal de Minas Gerais, Belo Horizonte, MG.

LUZ, C.R. Estudo cronológico sobre a raiva em Minas Gerais - no período de 1976 a 1986. 1988. 122f. Dissertação (Mestrado) - Escola de Veterinária, Universidade Federal de Minas Gerais, Belo Horizonte, MG.

OLIVEIRA, V.M.R. $O$ atendimento anti-rábico humano em Minas Gerais, de1999- 2004. 2005. 80f. Dissertação (Mestrado) - Escola de Veterinária, Universidade Federal de Minas Gerais, Belo Horizonte, MG.

PACHECO, C.A.C. Distribuição espaçotemporal da raiva em quirópteros em Minas Gerais, Brasil, 1969 a 2003. 2005. 48f. Dissertação (Mestrado) - Escola de Veterinária, Universidade Federal de Minas Gerais, Belo Horizonte, MG.

REIS, M.C.; COSTA, J.N.; PEIXOTO, A.P.C. et al. Aspectos clínicos e epidemiológicos da raiva bovina apresentados na casuística da clinica de bovinos. Rev. Bras. Saúde Prod. Anim., v.4, p.12-17, 2003. 
SILVA, M.C.P. Ocupação econômica da terra e a distribuição espacial da raiva bovina no Norte de Minas Gerais, Brasil (1982 a 1991). 1993. 61f. Dissertação (Mestrado) - Escola de Veterinária, Universidade Federal de Minas Gerais, Belo Horizonte, MG.

SILVA, J.A. Organização do espaço agrário e a distribuição da raiva bovina em Minas Gerais, 1976 a 1997. 1999. 199f. Tese (Doutorado) Escola de Veterinária, Universidade Federal de Minas Gerais, Belo Horizonte, MG.

SILVA, J.A.; MOREIRA, E.C.; HADDAD, J.P.A. et al. Distribuição temporal e espacial da raiva bovina em Minas Gerais, 1976 a 1997. Arq. Bras. Med. Vet. Zootec., v.53, p.263-272, 2001.

TADEI, V. A. Sistemática de quirópteros. $B$. Inst. Pasteur, v.1, p.3-15, 1996.

VALENTE, F.A.T.; AMARAL, L.B.S. Ocorrência de moléstia nos rebanhos bovinos do estado de São Paulo no triênio 1965/1967 Raiva nas regiões de Sorocaba, Presidente Prudente, Araçatuba e São José do Rio Preto. Biológico, v.38, p.25-29, 1972. 